\title{
IMBALANCE BETWEEN THYROID HORMONES AND THE DOPAMINERGIC SYSTEM MIGHT BE CENTRAL TO THE PATHOPHYSIOLOGY OF RESTLESS LEGS SYNDROME: A HYPOTHESIS
}

\author{
Jose Carlos Pereira Jr., ${ }^{\text {I Marcia Pradella-Hallinan, }}{ }^{\text {II Hugo de Lins PessoaI }}$
}

doi: $10.1590 / \mathrm{S} 1807-59322010000500013$

\begin{abstract}
Pereira Jr. JC, Pradella-Hallinan M, Pessoa HL. Imbalance between thyroid hormones and the dopaminergic system might be central to the pathophysiology of restless legs syndrome: a hypothesis. Clinics. 2010;65(5):547-54.

Data collected from medical literature indicate that dopaminergic agonists alleviate Restless Legs Syndrome symptoms while dopaminergic agonists antagonists aggravate them. Dopaminergic agonists is a physiological regulator of thyroid-stimulating hormone. Dopaminergic agonists infusion diminishes the levels of thyroid hormones, which have the ability to provoke restlessness, hyperkinetic states, tremors, and insomnia. Conditions associated with higher levels of thyroid hormones, such as pregnancy or hyperthyroidism, have a higher prevalence of Restless Legs Syndrome symptoms. Low iron levels can cause secondary Restless Legs Syndrome or aggravate symptoms of primary disease as well as diminish enzymatic activities that are involved in dopaminergic agonists production and the degradation of thyroid hormones. Moreover, as a result of low iron levels, dopaminergic agonists diminishes and thyroid hormones increase. Iron therapy improves Restless Legs Syndrome symptoms in iron deprived patients. Medical hypothesis. To discuss the theory that thyroid hormones, when not counterbalanced by dopaminergic agonists, may precipitate the signs and symptoms underpinning Restless Legs Syndrome. The main cause of Restless Legs Syndrome might be an imbalance between the dopaminergic agonists system and thyroid hormones.
\end{abstract}

KEYWORDS: Restless Legs Syndrome, RLS; RLS pathophysiology; Dopamine; TSH; CYP450.

Editors Note: This article opens a new section in which experienced authors will contribute a measure of speculative thought on apparently unrelated themes in medical practice.

\section{INTRODUCTION}

Dopamine (DA), when diminished, plays an important role in the pathophysiology of Restless Legs Syndrome (RLS) when decreased DA levels are a primary condition, or a secondary condition as is the case when low iron levels have been identified. It is known that DA agonists alleviate RLS symptoms, and there is medical evidence that DA depresses the thyroid axis, which is a concern in certain clinical settings.

The daily profile of thyroid-stimulating-hormone (TSH)

\footnotetext{
I Departamento de Pediatria, Faculdade de Medicina de Jundiaí - São Paulo/ SP, Brazil.

II Disciplina de Medicina e Biologia do Sono, Departamento de Psicobiologia, Universidade Federal de São Paulo - São Paulo/SP, Brazil.

Email: jhpessoa@terra.com.br

Tel: 5511 4586-4559

Received for publication on December 16, 2009

First review completed on January 08,2010

Accepted for publication on February 17, 2010
}

levels resembles the daily circadian intensity profile of symptoms of an RLS patient. Levels of TSH increase in the evening, as does the severity of RLS symptoms. To date, DA agonists have been considered to be the best therapy for RLS disturbances. In addition, the DA system modulates thyroid hormones $(\mathrm{TH})$. One mechanism by which $\mathrm{TH}$ is modulated by the DA system is through enhancement of the biochemical functions of the complex family of the cytochrome P450 (CYP450) enzymes. In addition, DA and DA agonists inhibit TSH secretion. The CYP450 enzymes are heme enzymes (all have iron) and the CPY450 enzyme superfamily is important for the biochemical degradation of TH. However, low iron levels diminish the quantity of CYP450 available to degrade TH. Several drugs that alleviate the symptoms of RLS are inducers of CYP450 activity, as induced CYP450 activity leads to enhanced degradation activity, causing TH levels to diminish.

RLS, also called Ekbom's syndrome, is a sensorimotor 
disorder that can be seen in approximately $1 \%$ to $15 \%$ of normal individuals; $5 \%$ to $10 \%$ of adults in Northern European population-based studies report RLS symptoms. ${ }^{1,2}$ The prevalence of RLS is increased with pregnancy, uremia, anemia, and rheumatoid arthritis; it is more common in women than men and its onset can occur at any age. ${ }^{3}$ It is now understood that considerably severe RLS can occur in up to $0.5 \%$ of children and $1 \%$ of adolescents. ${ }^{4}$ Significant variability in the severity of RLS is not uncommon. In the most severe cases, RLS may seriously impact a patient's quality of life..$^{1-7}$

The currently accepted definition of adult RLS from the "International Restless Syndrome Study Group (IRLSSG)" and the "International Classification of Sleep Disorders, second edition (ICSD-2)", are described below. All four features are necessary to make the diagnosis in adults and children. ${ }^{1,2,7}$

1) An urge to move the legs is usually accompanied or caused by uncomfortable and unpleasant sensations in the legs.

2) The urge to move or unpleasant sensations begin or worsen during periods of rest or inactivity, such as lying or sitting.

3) The urge to move or unpleasant sensations are partially or totally relieved by movements such as walking or stretching, as long as the activity continues.

4) The urge to move or unpleasant sensations are worse in the evening or night than during the day or only occur in the evening or night.

The supportive clinical features of RLS include a positive family history of RLS, periodic limb movements during sleep, and periodic limb movements during wakefulness. An additional supportive clinical feature is the "improvement of RLS symptoms with DA therapy". 1,2,7

\section{IRON LEVELS, TYROSINE HYDROXYLASE, CYTHOCROME P450, DA AND TH}

Among the associated clinical features of RLS, as stated by the IRLSSG, is a serum ferritin level $<50 \mu \mathrm{g} / \mathrm{L} .{ }^{7}$ Ferritin, an iron-binding protein is, at low levels, one of the most sensitive indicators of iron deficiency. Iron is a cofactor of tyrosine hydroxylase, a limiting enzyme in the synthesis of DA. Local iron deficiency in the substantia nigra could impair dopaminergic receptors or transporters. At the present time, there is robust evidence that low iron levels are one of the causative factors of RLS or of increasing its severity. ${ }^{8-10}$ Diminished DA function has a role in RLS symptoms, as can be inferred from the fact that DA agonists are beneficial to RLS patients, whereas antagonists are detrimental, and low iron levels are a putative cause of decreased DA function. ${ }^{8}$
The cytochrome P450 (CYP450) superfamily is another enzymatic complex that is down-regulated in response to iron scarcity. ${ }^{11}$ CYP450 is constituted by heme proteins and, thus, contains iron. ${ }^{12}$ In TH metabolism, the CYP450 complex is very important in the degradation of TH through what is called the "Phase 1 elimination of a drug". ${ }^{13}$ Drugs that inhibit CYP450 activity down-regulate the degradation of substances, such as TH, that are substrates of CYP450. The opposite occurs with drugs that induce CYP450 activity. ${ }^{13}$ The evidence described above allows us to infer that not only is iron deficiency a limiting condition for tyrosine hydroxylase activity, which is detrimental to RLS patients, but it also reduces CYP450 activity. It is important to also consider data previously published in the medical literature demonstrating that DA modulates $\mathrm{TH}$, that is, DA depresses the TH axis. ${ }^{14-16}$ Because the DA system enhances CYP450 activity and TH is a substrate for CYP450 enzymatic activity, it is possible to infer that one way that the DA system can modulate TH is via CYP450: the activation of CYP450 down-regulates TH. ${ }^{17}$ Furthermore, DA and DA agonists inhibit TSH secretion. ${ }^{16} \mathrm{CYP} 450$ isoforms are expressed in the liver and other peripheral organs, and also in glial cells and neurons in the brain ${ }^{18}$ (Figure 1).

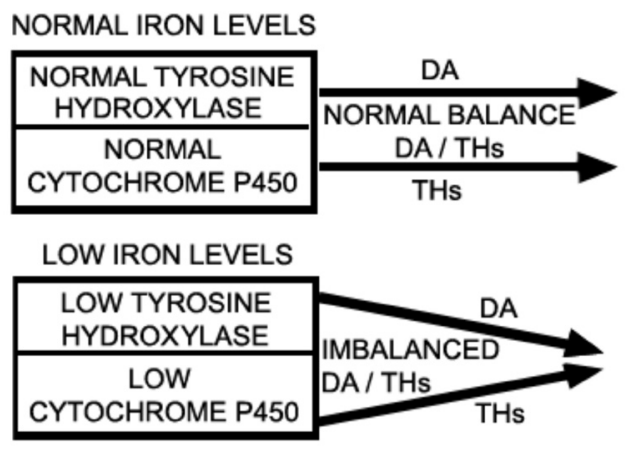

Figure 1 - A diagram showing the abnormal directions that DA and THs take under conditions of iron scarcity, and the resulting imbalance between DA and THs. Dopamine (DA). Thyroid Hormones (THs).

\section{DAILY TSH PROFILE AND CIRCADIAN ASPECTS OF RLS SYMPTOMS}

In the field of basic sleep science, an analysis of the daily TSH profile and its correlation with sleep phase is of great relevance. Also relevant is the comparison of the daily TSH profile and the propensity to sleep during times of the day that are not synchronized with sleep phase timing. The normal daily pattern of TSH secretion shows a circadian variation; circulating TSH concentrations are low and relatively stable during the daytime. TSH levels then begin an acrophase in the early evening, with a steep increase before sleep onset, and peak at around the beginning of the 
sleep period. In sequence, TSH levels progressively diminish throughout the sleep period until they reach their low daytime values. The circadian trough of TSH levels normally occurs between midday and $6 \mathrm{pm} .{ }^{19}$

Sleeping is not impossible out of the sleep phase, but the propensity to sleep usually varies significantly during the period between 7 am and $11 \mathrm{pm}$. The greatest propensity to sleep occurs in the mid-afternoon, which coincides with the circadian trough of TSH levels. The lowest propensity to sleep is in the evening during the steep circadian increase in TSH levels. ${ }^{20}$ Notably, the worsening of the symptoms of a typical RLS patient also occurs in the evening (fourth criterion of the IRLSSG) ${ }^{1,7}$ The parallel between the evening increase of TSH levels and the "worsening RLS symptoms" is of the utmost importance when considering the plausibility of the hypothesis proposed in this paper. The higher propensity to sleep when TSH levels are low, and the lower propensity to sleep when TSH levels are increasing could be a mere coincidence; however, considering that the effect of TH is to decrease the ability to sleep, even resulting in insomnia, and that diminished TH cause somnolence, ${ }^{21,22}$ it is likely that there is a causal relationship between TSH levels and RLS (Figure 2).

Analyzing plots of TSH values over a 24-hour period and the relationship of the curve to sleep phase or sleep propensity can contribute to our understanding certain circadian characteristics of the sleep-wake rhythm. These characteristics include the propensity to sleep, sleep itself, arousabilty from sleep, and voluntary or involuntary sleep deprivation. It has previously been held that the waveform of the daily profile values of TSH is influenced by sleep. ${ }^{19,23,24}$ This interpretation might not be totally accurate, as inference from the physiological and therapeutics effects of thyroid hormones (activity inducers) suggest that TSH levels influence sleep and not the contrary. In other words, the daily circadian waveform of thyrotropin could well be titled "The Thyroid Axis and Sleep", instead of "Sleep and the Thyroid Axis". The effects of TH are more likely to promote sleeplessness rather than sleepiness. ${ }^{21,22}$

Also relevant to this line of reasoning is the fact that the greatest propensity for sleep occurs during the circadian trough of the TSH waveform, from mid- to late-afternoon ${ }^{20}$. Could this heightened "propensity-for-sleep," rather than sleep itself, be responsible for the declining TSH values and the circadian trough of TSH? Could "sleep propensity" be a modulating condition of TSH? The possibility that the "low propensity for sleep" ${ }^{20}$ associated with the early evening is responsible for the steep increase in TSH levels (TSH surge) is also unlikely. Could "propensity-to-sleep" be considered a "function" that possibly modulates other functions?

The idea that sleep deprivation relieves the inhibitory effects of sleep on TSH levels ${ }^{23}$ is not correct. A more reasonable interpretation of the relationship between TSH level and sleep is that sleep deprivation is made possible by the elevation of $\mathrm{TH}$, not the reverse. As sleep deprivation is an abnormal condition, contrary to sleep-wake homeostasis, it is logical to infer that some function (or functions) must be executed to enable the curtailment of sleep. Considering the physiological and pharmacological profiles of $\mathrm{TH}$, they can be mainly considered as hormones inhibitors of sleep. In this light, TSH levels are important in modulating sleep propensity. During the biological day, the propensity for sleep is low in the late morning, ${ }^{20}$ a period when TSH levels are higher than during late afternoon. The fact that it is much more difficult to maintain wakefulness in the second half of the biological night, ${ }^{20}$ when TSH levels are lower than in the first half, needs to be factored into any understanding of these phenomena. From clinical RLS grounds it is well known that the best period for an RLS patient to sleep is in the early morning, which coincides with lower levels of TSH. It is also well known from clinical and pharmacology studies that sleep attack is a concerning side effect of DA therapy. ${ }^{3,5}$ A reasonable explanation for this fact is that DA therapy depresses $\mathrm{TH},{ }^{16}$ which are responsible for maintaining the alertness of wakefulness. It is equally important to analyze the interaction between TSH levels and sleep during the sleep phase. After sleep onset, TSH levels remain high during the night compared to daytime values. After reaching peak around sleep onset, TSH levels progressively decrease throughout the sleep phase towards low daytime values. ${ }^{19}$ In the case of sleep deprivation, this nocturnal decline is not observed..$^{23}$ Apparently there is a paradox with regard to the fact that TSH levels are higher during the sleep phase than during wakefulness, but this is likely not without explanation: at the onset of sleep, the circadian rhythm imposes its sleep drive and the individual starts sleeping; during sleep there is a dramatic decline in the sleeping person's alertness, but there remains an arousability condition inherent to the sleep state, critical for survival purposes. The physiological and clinical profiles of TH suggest that TH comprise the putative system that permits arousability from the sleep state.

From clinical settings, it is well known that hypothyroidism causes a low level of alertness and somnolence in patients. While the arousability condition inherent to the sleep state may involve additional systems, robust evidence in medical science leads us to posit that $\mathrm{TH}$ is very important to this biological phenomenon. The reason that TSH levels are depressed during wakefulness compared to the sleep phase can be logically explained by the following: the wakefulness state inherently maintains higher alertness, driven by circadian rhythmicity; thus necessitating lower TH levels. Some TH is needed, however, to prevent sleep from transforming into a coma state. It is 
of interest to mention that myxedema coma represents the extreme manifestation of severe hypothyroidism. ${ }^{22}$

\section{TSH LEVELS, DEEP NREM SLEEP, AND THE AL- PHA SLOW WAVE INDEX OF AROUSAL}

One important study used concomitant 10-minute TSH blood samplings and polysomnography in young volunteers. ${ }^{25}$ During normal sleep and recovery sleep after sleep deprivation, the descending slopes of TSH concentrations and the episodes of slow wave sleep (SWS) were selectively associated with each other. Conclusions drawn by the authors were that SWS appeared to be a key factor in the inhibitory effect of sleep on TSH secretion. However, the interpretation of the results of this work is questionable. There is no evidence that SWS inhibits TSH secretion, and the assumption that the decline of TSH values is a permissive condition for the occurrence of SWS seems more logical. A study examining the association between EEG spectral power and concomitant TSH values demonstrated a negative correlation between TSH fluctuations and SWS. ${ }^{19}$ This latter study concluded that the descending slopes of TSH levels help enable sleep to reach deep NREM stages. It was also demonstrated that the alpha slow wave index, an index of arousal derived from EEG, coincided with elevations of TSH concentrations. ${ }^{26}$ The overall nocturnal profile of TSH reflected the importance of high levels of TSH as an arousability factor. The higher the TSH profile, the higher the arousal index. Additionally, Brabant et al. ${ }^{19}$ and Gronfier et al. ${ }^{26}$ showed that TH does have a role in sleep modulation (Figure 2).

\section{SUMMARY OF THE INTERACTIONS BETWEEN DA, TH AND RLS.}

TH levels follow DA levels ${ }^{14-16}$ Thus, the depressing effects of DA on TH are a concern in certain clinical settings. ${ }^{27-29}$ Thyroid-releasing hormone (TRH) regulates TSH synthesis by stimulating the transcription and translation of the TSH $\beta$-subunit gene; dopamine inhibits this process. ${ }^{30}$ Anti-DA drugs such as metoclopramide aggravate the symptoms of RLS, ${ }^{17}$ while DA agonists diminish RLS symptoms. ${ }^{31-35}$ Reduced levels of iron diminish the DA system, which in turn aggravates RLS..$^{10-12,22} \mathrm{TH}$ axis heightens during sleep deprivation, as demonstrated in voluntary sleep deprivation. ${ }^{23}$ Sleep deprivation, in turn, is well known to aggravate RLS symptoms. ${ }^{5}$ Some of the effects of increased TH, such as hyperthyroidism, resemble some symptoms of RLS patients..$^{21,22}$ Opioids, the first class of drugs used to treat RLS, ${ }^{3,5}$ are also known to depress the TH axis. ${ }^{36,37}$ Some drugs that are effective against RLS

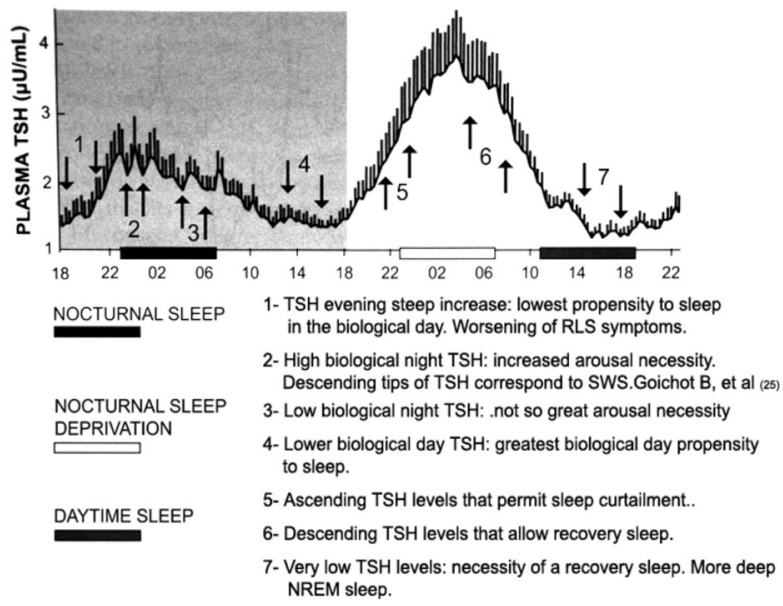

Figure 2 - depicts the proposed relationship between TSH levels with sleep propensity and sleep. Modified from Pannain and Van Cauter ${ }^{24}$. "Mean 24-hour profile of plasma TSH during a 53-hour period including 8 hours of nocturnal sleep (black bar), 28 hours of continuous wakefulness (nocturnal hours: white bar), and 8 hours of daytime sleep (black bar at the right). Subjects were young healthy men. The shadow area corresponds to normal 24-hour conditions. The vertical bar at each time point represents the standard error of the mean (SEM) ..." (Pannain S, Van Cauter E. Sleep Medicine Clinics 2007; 2: 147-159).

symptoms, such as carbamazepine, phenobarbital, valproate, and primidone are inducers of CYP450 enzyme activity. ${ }^{38}$ Selective serotonin reuptake inhibitors, neuroleptics, tricyclic antidepressants, and antihistamines are all CYP450 inhibitors, ${ }^{39}$ and they all worsen the symptoms of an RLS patient. ${ }^{3,5}$ It is unlikely that the facts above be mere coincidence (Figure 3 ).
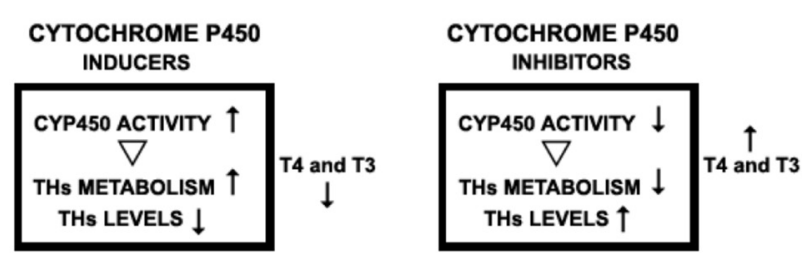

Figure 3 - A diagram showing the effects of CYP450 inducers or inhibitors on TH metabolism and the final results of these pharmacological actions on T4 and T3 levels. Thyroid hormones (THs); Thyroxine (T4); Triiodothyronine (T3).

\section{RLS IN PREGNANCY}

RLS is more common in pregnant women than in nonpregnant women, ${ }^{5}$ and endocrinological studies in pregnancy have revealed that $\mathrm{TH}$ are up regulated during pregnancy. ${ }^{40}$ This phenomenon supports the hypothesis proposed here, although it is possible that other conditions might be involved in the greater prevalence of RLS during pregnancy. A recent work showed elevated estradiol plasma levels in RLS pregnant women ${ }^{41}$ The authors raised the possibility 
that the augmented estradiol could be a causative factor for RLS. However, as there is no evidence of elevated estradiol in non-pregnant RLS patients, it must be inferred that the causality of elevated estradiol on RLS in pregnancy, is, at best, indirect. The parallel between the augmented hormones $\mathrm{TH}$ and estradiol in pregnancy deserves comment. One hormonal effect of estradiol is to increase levels of TH. ${ }^{42}$ Thus, one possible cause of higher TH levels in pregnancy is the higher levels of estradiol, which suggests that estradiol may indirectly cause symptoms of RLS by increasing TH. If these augmented TH levels are not counterbalanced by the DA system, RLS would appear. It is known that estradiol is a CYP450 inhibitor, and it also is a condition for the thyroid binding globulin to increase. ${ }^{43,44}$

\section{HYPERTHYROIDISM AND RLS SYMPTOMS}

An interesting case report from Tan and colleagues (2004) illustrates the correlation between TH levels and low iron levels. A hypothyroid patient with low serum ferritin treated with L-thyroxine developed signs and symptoms of RLS. Upon challenge and withdrawal of L-thyroxine, there was a significant change in the International Restless Legs Syndrome Study Group severity score (26/40 to 6/40), the periodic limb movements in sleep (PLMS) index (20/hour to $10 /$ hour), the number of arousals due to PMLS (59 to 22), sleep efficiency (74 to $85 \%$ ), and other biochemical parameters ${ }^{45}$. As many patients with normal iron levels have RLS, one cannot assume that elevated TH and low iron levels are the main dysregulations that cause RLS. It is, however, possible to infer that this abnormal correlation precipitates or aggravates RLS symptoms. L-thyroxine is usually administered to hypothyroid patients in the morning, which essentially decouples TH levels from the circadian biological clock. Therefore, it is not always possible to observe the fourth diagnostic criterion of the IRLSSG ( worsening of symptoms in the evening and night ).

The greater prevalence of RLS in patients with hyperthyroidism over healthy subjects could represent the ultimate proof of the importance of TH on the genesis of RLS. This assumption might not be that straightforward since there are several factors to be considered. For example, one must also take into account the modulation of $\mathrm{TH}$ by the DA system, which cannot be assumed to always be impaired. Based on the data we gathered, it is reasonable to conclude that the balance between TH and the DA system is what counts most. It is their relative values, one in relation to another, that are important.

Despite the dearth of studies on concomitant hyperthyroidism and RLS, an important study by Tan and colleagues ${ }^{46}$ emphatically demonstrated that a close relationship between hyperthyroidism and RLS symptoms does exist. This study examined 125 hyperthyroid patients, 21 hypothyroid patients, and 434 normal subjects (control group). None of the thyroid patients were diagnosed as having RLS as defined by the IRLSSG, ${ }^{7}$ that is, no patients met all four criteria for the RLS diagnosis. The authors of the study also found that RLS has a low prevalence in Singapore where the study took place, as only one subject amongst the 434 subjects of the control group fulfilled all the criteria for diagnosis of RLS ( $0.2 \%)$. Although any case of RLS has been found amongst the hyperthyroid patients, the authors observed RLS-like symptoms corresponding to the three criteria of the IRLSSG in a significant number of the patients. In normal subjects, $4 / 434(0.9 \%)$ met the first three diagnostic criteria of RLS, as did $1 / 21$ hypothyroid patients (4.8\%), and $11 / 125$ hyperthyroid patients $(8.8 \%))^{46}$ These numbers are significant as they seem to substantiate the claim that elevated TH is an inciting condition for RLS-like symptoms.

In accordance with the hypothesis dealt with in this paper, it is these authors' belief that the fourth criterion that defines the RLS diagnosis is a consequence of the circadian increase of the TH axis in the evening. In Graves' disease, the thyroid gland is dysregulated and TH levels do not follow the circadian TH rhythm of a typical normal subject. ${ }^{47}$

One can assume that a hyperthyroid patient presenting with three of the four criteria ${ }^{46}$ that define the disease might not have the disease but rather only symptoms of it. The results from the treatment of the hyperthyroid patients ${ }^{46}$ were noteworthy. Four patients among the 12 with RLS-like symptoms reported complete resolution of their complaints (33\%): three hyperthyroid to euthyroid, and one hypothyroid to euthyroid. The others reported some improvement in their symptoms. ${ }^{46}$ Only these results, pointed out by TAN and colleagues are significant per se. However, 4 out of 11 patients who were hyperthyroid at the beginning of the treatment and reported some improvement after treatment, were still biochemically hyperthyroid when the results of the treatment were tabulated. Logic permits us to suppose that the results of the treatment would have even been better if the conclusions about the treatment had been drawn after these four hyperthyroid patients had become euthyroid. Moreover, elevated TH is not the only factor that can cause RLS, but it is the inciting condition for the syndrome, not the syndrome on its own. Concerning the relationship between the DA system and thyroid status, the reader is encouraged to review the work of Strawn JR and colleagues. ${ }^{48}$

\section{FINAL REMARKS}

The RLS augmentation phenomenon, which is a concern when treating a patient with DA agonists,, 3 could be partly 
explained by the common biofeedback mechanism that occurs when a hormone is being artificially diminished. Tolerance for a drug that is both an inducer and substrate of CYP450 could also explain some of the characteristics of the augmentation phenomenon. For example, tolerance to carbamazepine, ${ }^{49}$ which is also an effective treatment for RLS, is common. This drug, an inducer and substrate of CYP450, slowly causes tolerance because the induction of CYP also increases the speed with which carbamazepine itself is metabolized, rendering it less effective at inducing metabolism of TH. Notwithstanding, we do agree that there is not, as yet, a cognizance of the real causes of the augmentation phenomenon. Thus, it is difficult to explain why RLS symptoms start earlier in the day after the RLS augmentation phenomenon has been initiated.

RLS is more common in older patients who are known to have a slower metabolism of TH compared to younger subjects. Hypothyroidism in older patients usually requires lower doses of thyroid hormone replacement. ${ }^{50}$ In light of the hypothesis of this work, there is a reasonable possibility that the slower metabolism of TH in the older subjects makes them more susceptible to RLS. In addition, subclinical hyperthyroidism is also more common (2\%) in elderly subjects. ${ }^{50}$

"Growing pains" are common in childhood RLS, ${ }^{51}$ and some adult patients also complain of pain concomitant with other classical symptoms of RLS in the legs. ${ }^{3,5}$ A very important study by Ondo and colleagues (2000) demonstrated that the dopaminergic system in rats sends long projections from the midbrain into the spinal cord, which influences the gating of peripheral nociceptive input. ${ }^{52}$ With the profuse data demonstrating the tight interactions between $\mathrm{TH}$ and DA, it seems plausible that TH mediates these nociceptive inputs and that pain ensues when these inputs are not counterbalanced by DA. Growing pains occur mainly in the evening or night when TH levels are higher; they usually do not occur during the day when TH levels are lower.

Rye and Freeman reported that DA synthesis, release, and signaling peak early during the active period and reach their nadir in the sleep phase. ${ }^{53} \mathrm{DA}$ allows the gating of inputs via alteration of membrane properties and specific ion conductances. ${ }^{54}$ Rye and Freeman explain that the physiological effects of the DA system are best characterized as "neuromodulatory," rather than eliciting excitatory or inhibitory postsynaptic potentials. ${ }^{53}$ From the considerations about RLS clinical and physiopathology issues described above, it is possible to infer that the DA system also has a hormonal modulatory effect on $\mathrm{TH}$.

The hypothesis we presented refers only to primary RLS cases and those that are secondary to iron deficiency and pregnancy, which constitute the great majority of RLS patients. For these clinical situations, we have found strong evidence to support the hypothesis. We did not attempt to theorize about the pathophysiology of other secondary cases of RLS because we believe that doing so would be only speculation, in light of the few existent data on secondary RLS cases at this moment. We have also chosen not to address "Periodic Limb Movement in Sleep" for the same reason.

This theoretical study attempts to establish a unified theory for RLS pathophysiology through a review of the current knowledge on this topic. A hypothesis is rendered null and void if it is not supported by experimentation to validate it. Studies focusing on the TH axis and DA system in patients suffering from RLS might teach us more about this very serious human disease. A more detailed understanding of the importance of the cytochrome P450 enzyme system in RLS is warranted, as are studies of the genetics of the cytochrome P450 system. Moreover, iron deprivation might not be a concern restricted to RLS.

Another issue raised by this paper is whether deviations of the normality of TH axis and DA system interaction might actually influence the quality of sleep in sleep disturbances other than RLS. Thus, RLS could be considered a paradigmatic disease for other sleep disturbances.

We are much indebted to Dr Arthur S. Walters, Professor of Neurology Vanderbilt School of Medicine, Nashville, Tenesee, USA, for help criticism of the manuscript and kindly attention.

We also thank to Dr Thales M. Pereira for drawing the figures of this manuscript.

\section{REFERENCES}

1. American Academy of Sleep Medicine. International classification of sleep disorders, sec. edition. Diagnostic and coding manual. Wetchester, Illinois: American Academy of Sleep Medicine, 2005.

2. Allen R, Picchietti D, Hening W, Tremkwalder C, Walters AS, Montplaisir J. Restless legs syndrome: diagnostic criteria, special considerations, and epidemiology. A report from the restless syndrome diagnosis and epidemiology workshop at the National Institutes of Health. Sleep Med. 2003;4:101-19.
3. Lee Chiong TL, Sateia M, Carskadon M, Eds. Sleep Medicine. Philadelphia, PA: Hanley\&Belfus (Elsevier), 2002.

4. Picchietti D, Allen RP, Walters AS, Davidson JE, Myers A, FeriniStrambi L. Restless legs syndrome: prevalence and impact in children and adolescents. The Peds Rest Study. Sleep. 2006;29:29A70.

5. Earley C. Clinical practice. Restlesslegs syndrome. N Engl J Med. 2003;348:2103-9. 
6. Ekbon K. Restless legs: a clinical study. Acta Med Scand. 1945;158:1122.

7. Walters AS. Group organizer and Correpondent International Restless Legs Syndrome Study Group. Towards a better definition of the restless legs syndrome. Mov Disord. 1995;10:634-42.

8. St O' Keeffe, Gavin K, Lavan JN. Iron status and restless legs syndrome in the elderly. Age Ageing. 1994;23:200-03.

9. J Krieger, C Schroeder. Iron status and restless legs syndrome. Sleep Medicine Reviews. 2001;5:277-86.

10. Allen R. Dopamine and iron in the pathophysiology of restless legs syndrome. Sleep Med 2004;4:385-91.

11. Huebers HA. Non hematological manifestations of iron deficiency. In Bo Lonnerdal, editor, Iron Metabolism in Infants, CRC Press, 1990;pgs 63-72.

12. Dallman PR, Goodman Jr. The effects of iron deficiency on the hepatocyte. A biochemical and ultrastructural study. JCB. 1971;48:7990.

13. Cupp MJ, Tracy TS. Cytochrome P450: New nomenclature and Clinical Implications. American Family Physician. 1998;57:117-26.

14. Feek CM, Sawers JSA, Brown NS, Seth J, Irvine WJ, Toft AD. Influence of thyroid status on dopaminergic inhibition of thyrotropin and prolactin secretion: evidence for an additional feedback mechanism in the control of thyroid hormone secretion. J Clin Endocrinol Metabol. 1980;51:5859 .

15. Scanlon MF, Weetman AP, Lewis M, Pourmand M, Rodrigues A, Weightman DR. Dopaminergic modulation of circadian thyrotropin rhythm and thyroid hormone levels in euthyroid subjects. J Clin Endocrinol Metabol. 1980;51:1251-6.

16. G Delitala. Dopamine and TSH secretion in man. The Lancet. 1977;310:760-1.

17. Wojcikowski J, Gocen Biowska K, Wladystawa AD. Regulation of liver cythocrome $\mathrm{P} 450$ by activation of brain dopaminergic system. Biochem Pharmacol. 2008;76:258-67.

18. Meyer RP, Gehlhaus M, Knoth R, Volk B. Expression and function of cytochrome p450 in brain drug metabolism. Curr Drug Metab. 2007;8:297-306

19. Brabant G, Prank K, Ranft U, Schuermeyer TOF, Wagner H, Hauser $\mathrm{B}$, et al. Physiological regulation of circadian and pulsatile thyrotropin secretion in normal man and woman. J Clin Endocrinol Metab. 1990;70:403-9.

20. P. Lavie. Sleep-Wake as a biological rhythm. Annual Review of Psychology. 2001; pg277.

21. Guyton AC.Textbook of Medical Physiology. 7th Ed. W.B. Saunders Company, 1981.

22. Farwell AP, Braverman LE. Thyroid and Antithyroid Drugs. In Hardman JG, Limbird LE, Gilman AG, editors. Goodman and Gilman's The Pharmacological Basis of Therapeutics. Tenth edition. McGraw-Hill, New York, 2001;1563-96.

23. Parker DC, Rossman LG, Pekary AE, et AL. Effect of 64-hour sleep deprivation on the circadian waveform of thyrotropin (TSH): further evidence of sleep-related inhibition of TSH release. J Clin Endocrinol Metab. 1987;64:157-61.
24. Pannain S, Van Cauter E. Modulation of Endocrine Function by SleepWake Homeostasis and Circadian Rhythmicity. Sleep Medicine Clinics. 2007;2:147-59.

25. Goichot B, Branderberg G, Saini J, Wittersheim G, and Follenius M. Nocturnal plasma thyrotropin variations are related to slow-wave sleep. J Sleep Res. 1992; 1:186-90.

26. Gronfier C, Luthringer R, Follenius M, Schaltembrand N, Macher JP, Muzet A, et al. Temporal link between plasma thyrotropin levels and electroencephalographic activity in man. Neurosci Lett. 1995;200:97-100.

27. Kaptein EM, Spencer CA, Kamiel MB, and Nicoloff JT. Prolonged dopamine administration and thyroid hormone economy in normal and critically ill subjects. J. Clin Endocrinol Metab. 1980;51:387-393.

28. Van den Berghe G, de Zeghert F, Lauwers P. Dopamine and the sick euthyroid syndrome in critical illness. Clin Endocrinol.1994;41:731-7.

29. Debayeve YA, Van den Berghe GH. Is there still a place for dopamine in the modern intensive care unit? Anesth Analg. 2004;98:461-8.

30. Shupnik MA, Greenspan SL, Ridgway EC. Transcriptional regulation of thyrotropin subunit by thyrotropin-releasing hormone and dopamine in pituitary cell culture. J. Biol. Chem 1986;261:675-9.

31. Akpinar S. Restless legs syndrome treatment with dopaminergic drugs. Clin Neuropharmacol. 1987;10:69-79.

32. Walters AS, Ondo WG, Dreykluft T, GrunsteinR, Lee D, Sethi K. Ropinirole is effective in the treatment of restless legs syndrome: TREAT RLS 2: a 12 -week, double-blind, randomized, parallel-group, placebo-controlled study. Mov Disord. 2004;19:1414-23.

33. Walters A, Hening W, Kavey N, Chokroverty S, Sidro-Frank S. A Double-blinded randomized cross-over trial of bromocriptine and placebo in restless legs syndrome. Ann Neurol. 1988;24:455-8.

34. Scanlon MF, Weightman DR, Shale DJ, Mora B, Heath M, Snow MH, et al. Dopamine is a physiological regulator of Thyrotropin (TSH) in normal man. Clin Endocrinol. 1978;10:7-15.

35. Filippi L, Pezzati M, Cecchi A, Poggi C. Dopamine infusion: A possible cause of undiagnosed congenital hypothyroidism in preterm infants. Pediat Crit Care Med. 2006;7:249-51.

36. Zhang GF, Tang YL, Smith AK, Zheng-gi L, Sheng LX, Yong C, et al. Alterations in pituitary-thyroid axis function among opioids-dependent subjects after acute and protracted abstinence. Addiction biology. 2009;14:310-4.

37. Ruzsas C, Mess B. Opioidergic regulation of thyroid activity: possible interference with the serotoninergic system. Psychoneuroendocrinology. 1983;8:89-94

38. McNamara JO. Drugs effective in the therapy of the epilepsies. In Hardman JG, Limbird LE, Gilman AG, editors, The Pharmacological Basis of Therapeutics, tenth edition, 2001. McGraw-Hill, New York.

39. Indiana University Division of Clinical Pharmacology. Cytochrome P450 Drugs Interaction Table. Version. 5;2009.

40. Glinoer D. The regulation of thyroid function in pregnancy: Pathway of endocrine adaptation from physiology to pathology. Endocrine Reviews. 1997;18:404-33.

41. Dzaja A, Wehrle R, lancel M, Pollmacher T. Elevated estradiol plasma levels in women with restless legs during pregnancy. Sleep. 2009;32:16974. 
42. Sanger N, Stahlberg S, Manthey T, Mittman K, Mellinger U, Lange E, et al. Effects of an oral contraceptive containing $30 \mathrm{mcg}$ ethynil estradiol and $2 \mathrm{mg}$ dienogest on thyroid hormones and androgens parameters: conventional VS. extended cycle use. Contraception. 288;77:420-5.

43. Martucci CP, Fishman J. P450 enzymes of estrogen metabolism. Pharmacol Ther. 1993;57:237-57.

44. Lee AJ, Cai MX, Thomas PE, Conney AH, Zhu BT. Characterization of the oxidative metabolites of 17 beta-estradiol and estrone formed by 15 selectively expressed human cytochrome p450 isoforms. Endocrinology. 2003;144:382-98

45. Tan EK, Ho SC, Koh L, Pavanni R. An urge to move with L-thyroxine: clinical, biochemical, and polysomnographic correlation. Mov Disord. 2004;19:1365-7.

46. Tan EK, Ho SC, Eng P, Loh L, Koh L, Lum S, et al. Restless legs symptoms in thyroid disorders. Parkinsonism and Related Disorders. 2004; 10:149-51.

47. Schuppert F, Diegelmann B, Geest T, Wagner TO, von-zur Muhlen A. Loss of variability in Graves' disease stimulatory TSH-receptors antibodies bind to the TSH-receptor in a continued non pulsatile and non-chaotic fashion. Chronobiologia. 1994;21:21-32.

48. Strawn JR, Ekhator NN, D'Souza BB, Geracioti TD, Geracioti JR Pituitary-thyroid state correlates with central dopaminergic and serotoninergic activity in healthy humans. Neuropsychobiology. 2004;49:84-7.
49. Rang HP, Dale MM, Ritter JM, Flower RJ. Rang and Dale's Pharmacology, $6^{\text {th }}$ edition, 2008. Elsevier, USA.

50. Greenspan SL, Resnick NM. Geriatric Endocrinology in Greenspan'S Basic \& Clinical Endocrinology. Gardner DG and Shoback D, editors. McGraw-Hill, 2007, $8^{\text {th }}$ edition. Pgs 844-67.

51. Walter AS. Is there a subpopulation of children with growing pains who really have restless legs syndrome? A review of the literature. Sleep Med. 2002b;3:93-8.

52. Ondo WG, He Y, Rajasekaran S, Le WD. Clinical correlates of 6-hydroxydopamine injections into A11 dopaminergic neurons in rats: a possible model for restless legs syndrome. Mov. Disord. 2000;15:154-8.

53. Rye DB, Freeman AAH. Dopamine in Behavioral State Control, in Monti JM, Pandi-Perumal SR, and Sinton CM, editors: Neurochemistry of Sleep and Wakefulness. Cambridge University Press, Cambridge, 2008. Pgs 179-223.

54. Nicola S, Surmeir J, Malemka R. Dopaminergic modulation of neuronal excitability in the striatum and nucleus acumbens. A. Rev. Neurosci. 2000;23:185-215. 